\title{
Are the E-type asteroids (2867) Steins, a target of the Rosetta mission, and NEA (3103) Eger remnants of an old asteroid family?`
}

\author{
S. Fornasier ${ }^{1,2}$, F. Marzari $^{3}$, E. Dotto ${ }^{4}$, M. A. Barucci ${ }^{1}$, and A. Migliorini ${ }^{5}$ \\ 1 LESIA, Observatoire de Paris, 92195 Meudon Principal Cedex, France \\ e-mail: sonia.fornasier@obspm.fr \\ 2 Université de Paris 7 Denis Diderot, 10 rue Alice Domon et Leonie Duquet, 75013 Paris, France \\ 3 Dipartimento di Fisica, Università di Padova, via Marzolo 8, 35131 Padova, Italy \\ 4 INAF, Osservatorio Astronomico di Roma, via Frascati 33, 00040 Monteporzio Catone, Roma, Italy \\ 5 IASF - INAF, via del Fosso del Cavaliere 100, 00133 Roma, Italy
}

Received 3 August 2007 / Accepted 7 September 2007

\section{ABSTRACT}

\begin{abstract}
Aims. We have investigated the spectral properties of the E-type asteroids 2867 Steins, a main belt object target of the Rosetta mission, and 3103 Eger, a near Earth asteroid. The strong spectral similarity between these two objects suggests a possible common origin in spite of their presently different orbits. We explore the possibility that Steins and Eger are both remnants of an old asteroid family, the outcome of the breakup of a parent body at about 2.36 AU. Eger possibly moved into an Earth-crossing orbit driven by the Yarkovsky effect and resonances.

Methods. Low resolution spectroscopy in the visible range was carried out with the $3.5 \mathrm{~m}$ New Technology Telescope (NTT) of the European Southern Observatory. We used the numerical integrator SWIFT-RMVSY, which takes into account the diurnal and seasonal Yarkovsky effect, to simulate the dynamical evolution of fictitious family fragments.

Results. The spectra of Steins and Eger are very similar, and both show an absorption feature centered at $0.49 \mu \mathrm{m}$ typical of the E[II] subgroup. They are peculiar among the subgroup because of the deep absorption feature and steep spectral slope. They may be members of an old eroded asteroid family which formed close to the present location of Steins. Numerical orbital integrations show that there is a dynamical pathway between the present orbit of Steins, possibly the largest remnant of the family, and Earth-crossing orbits like that of Eger.
\end{abstract}

Key words. minor planets, asteroids - techniques: spectroscopic - methods: observational - methods: numerical

\section{Introduction}

The E-type asteroids are a small population of bodies that have experienced high thermal evolution and are thought to be the parent bodies of enstatite achondrites meteorites (also known as the aubrites). Their surface composition seems to be dominated by iron-free or iron-poor silicates such as enstatite, forsterite or feldspar, and their spectra resemble the aubrite meteorite spectra (Gaffey et al. 1992; Clark et al. 2004). Initially, they were believed to be featureless and with a spectral behaviour similar to the M- and P-type asteroids, from which they were distinguished only on the basis of the albedo, the highest of any asteroid class (around 0.4-0.6). Since 1995 some absorptions bands, both in the visible and near infrared region, have been identified in their spectra, and three different surface mineralogies have been suggested to be present inside the E-type population (Clark et al. 2004; Gaffey \& Kelley 2004). Following the Gaffey \& Kelley (2004) classification scheme, we observe 3 subgroups. $\mathrm{E}[\mathrm{I}]$ asteroids do not present absorption features, but a slightly reddish slope in the visible spectral range. They might be the parent bodies of the aubrite meteorites, and they must have experienced temperatures higher than $1400^{\circ} \mathrm{C}$. E[II]

* Based on observations carried out at the European Southern Observatory (ESO), La Silla, Chile, ESO proposal 076.C-0047 and 078.C-0115. members present a strong absorption at $\sim 0.49 \mu \mathrm{m}$ and occasionally at $\sim 0.90-0.96 \mu \mathrm{m}$. These features are possibly due to the calcium sulfide mineral oldhamite (present in highly reduced assemblages such as aubrites). E[III] members are characterised by a flat or reddish spectral curve with a well defined band centered at $\sim 0.9 \mu \mathrm{m}$, due to enstatite pyroxene containing $\mathrm{Fe}^{2+}$. Some asteroids having the $0.9 \mu \mathrm{m}$ band show an absorption feature at $1.8 \mu \mathrm{m}$ (Clark et al. 2004). The asteroids of this subgroup are thought to come from oxidised parent bodies that underwent extensive reduction during their igneous processing.

In this work, we present new visible spectra of the two E-type asteroids 2867 Steins and 3103 Eger which suggest a strong "genetic" relation, in a mineralogical sense, between the two bodies.

2867 Steins is the first target that will be explored by the Rosetta mission with a flyby on 5 September 2008. The closest approach will take place at $1745 \mathrm{~km}$, with a relative velocity of $8.7 \mathrm{~km} \mathrm{~s}^{-1}$. The asteroid is located at $2.36 \mathrm{AU}$, it has a rotational period of $6.052 \pm 0.007 \mathrm{~h}$ (Kuppers et al. 2007), and an estimated diameter of $4.8-6.0 \mathrm{~km}$.

Barucci et al. (2005) presented the first spectroscopic observations of 2867 Steins, finding a spectral behaviour similar to the E-type asteroid 64 Angelina and characterised by a strong $0.49 \mu \mathrm{m}$ absorption band, attributed to the presence of sulfides such as oldhamite. The classification of Steins as an igneous E-type asteroid was strengthened by its high albedo value $(0.45 \pm 0.10)$ determined with polarimetry (Fornasier et al. 2006). 
Table 1. Observational conditions: $T_{\exp }$ is the exposure time in seconds, air. is the mean airmass of each object during the exposure time.

\begin{tabular}{llcccc}
\hline \hline Object & Night & $\begin{array}{c}\mathrm{UT}_{\text {start }} \\
(\mathrm{hh}: \mathrm{mm})\end{array}$ & $\begin{array}{c}T_{\exp } \\
(\mathrm{s})\end{array}$ & $\begin{array}{c}\text { Air. } \\
\text { Solar Analog } \\
\text { (air.) }\end{array}$ \\
\hline 2867 Steins & 25 May 04 & $23: 45$ & 1200 & 1.41 & la102-1081 (1.20) \\
2867 Steins & 13 Aug. 05 & $07: 10$ & 780 & 1.05 & HD 1835 (1.07) \\
3103 Eger & 20 Jan. 07 & $06: 56$ & 780 & 1.31 & la98-978 (1.35) \\
\hline
\end{tabular}

Barucci et al. (2007), analysing the spectral data in the 5-38 $\mu \mathrm{m}$ wavelength range obtained with the Spitzer Space Telescope, found that the emissivity spectrum of Steins is similar to that of enstatite and aubrite meteorites, confirming the E-type classification.

3103 Eger is an Apollo Near Earth asteroid (NEA). It was the first NEA classified as E-type. Its orbit has a semimajor axis of 1.4 AU, eccentricity of 0.35 and inclination of about 21 degrees. The orbital intersection between 3103 Eger and the Earth seems to be a favourable source of the aubrite meteorites (Gaffey et al. 1992; Binzel 1995). Its rotational period is of $5.705 \mathrm{~h}$, and the estimated albedo is 0.63 (Veeder et al. 1989).

\section{Observations and data reduction}

The data presented in this work were obtained in the visible range during 3 runs at the ESO-NTT telescope, on May 2004, August 2005 and January 2007, in the framework of a spectroscopic survey of E-type asteroids. The conditions of the observations are reported in Table 1.

The spectra were acquired using the EMMI instrument, equipped with a $2 \times 1$ mosaic of $2048 \times 4096$ MIT/LL CCD with square $15 \mu \mathrm{m}$ pixels. We used the grism \#1 (150 gr/mm) in RILD mode to cover the wavelength range $0.41-0.94 \mu \mathrm{m}$, with a dispersion of $3.1 \AA / p x(200 \AA / \mathrm{mm})$ at the first order. The spectra were taken through a 2 arcsec wide slit, oriented along the parallactic angle. Several solar analog stars were observed with the same instrumental set up during each night.

The data were reduced using standard procedures with the software packages Midas and IDL as described in Fornasier et al. (2004): subtraction of the bias from the raw data, flat-field correction, cosmic rays removal, sky subtraction, collapsing the two-dimensional spectra to one dimension, wavelength calibration and atmospheric extinction correction.

The reflectivity of each asteroid was obtained by dividing its spectrum by that of the solar analog star closest in time and airmass to the object. The reflectance spectrum was normalised to $0.55 \mu \mathrm{m}$ and smoothed with a median filter technique.

We evaluate the asteroids' spectral slope in the $0.55-0.80 \mu \mathrm{m}$ range using a standard least square technique.

\subsection{Steins}

We obtained two visible spectra of Steins on 25 May 2004 and 13 August 2005 (Fig. 1). Both spectra show the sharp $0.49 \mu \mathrm{m}$ absorption band, probably associated with the presence of oldhamite, and typical of the E[II] subgroup following the Gaffey \& Kelly (2004) classification scheme. The band is centered at $4943 \pm 22 \AA$ in the spectrum of May 04 and $4954 \pm 20 \AA$ in the August 05 one. The absorption feature is extended from around 0.44 to $0.55 \mu \mathrm{m}$, with a depth of $12 \%$ compared to the continuum. We do not clearly detect any feature in the $0.9 \mu \mathrm{m}$ region that is often associated with the $0.49 \mu \mathrm{m}$ band.

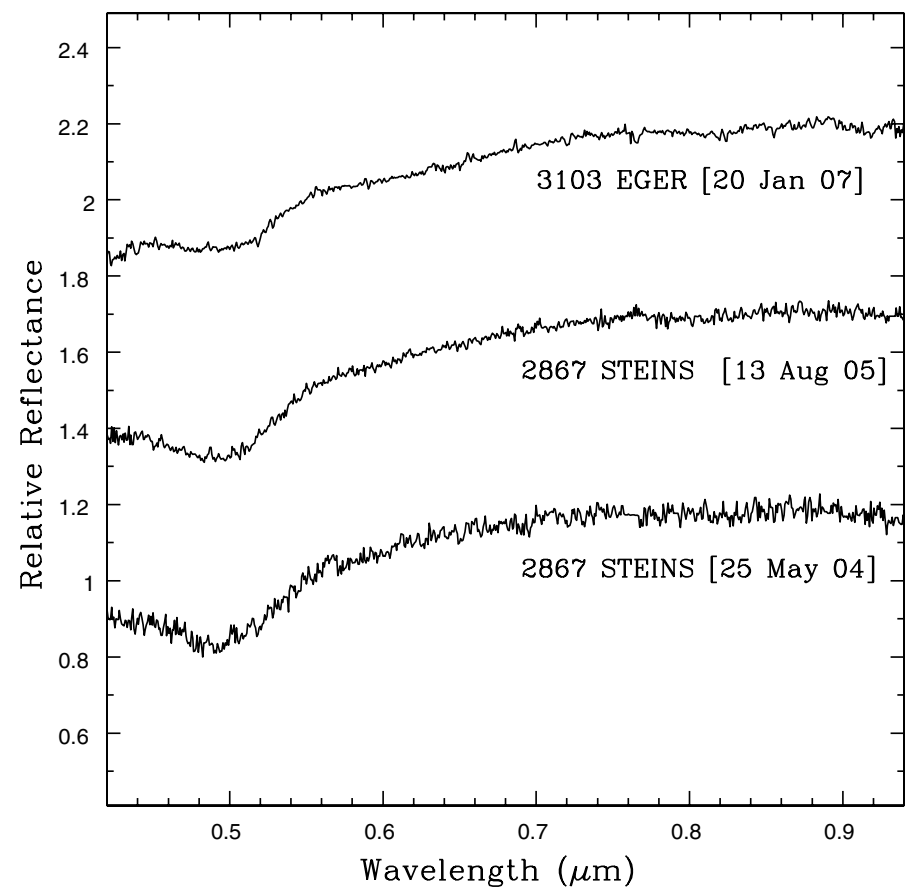

Fig. 1. Visible spectra of 2867 Steins, target of the Rosetta mission, and of the NEA 3103 Eger.

Barucci et al. (2005) reported the presence of a faint feature at about $0.96 \mu \mathrm{m}$ and a flat and featureless behaviour beyond $1 \mu \mathrm{m}$.

The spectral slope of the two spectra is comparable within the error bars. It is of $6.93 \pm 0.65 \% / 10^{3} \AA$ for the May 2004 data and of $7.62 \pm 0.64 \% / 10^{3} \AA$ for the August 2005 observations.

\subsection{Eger}

We observed 3103 Eger on 20 January 2007. The spectrum shows an absorption band centered at $4985 \pm 25 \AA$ (Fig. 1), extended from around 0.44 to $0.55 \mu \mathrm{m}$ and of $\sim 8.5 \%$ depth compared to the continuum. The slope is of $7.40 \pm 0.53 \% / 10^{3} \AA$, very similar to the value $\left(7.3 \% / 10^{3} \AA\right)$ previously determined by Fornasier \& Lazzarin (2001). Beyond $0.8 \mu \mathrm{m}$ the spectrum becomes altmost flat, and we do not clearly detect any absorption band at $0.9 \mu \mathrm{m}$, while Burbine et al. (1998) identified on their spectrum a faint absoption that extends past $0.92 \mu \mathrm{m}$.

The visible spectrum of Eger is typical of the E[II] subgroup following the Gaffey \& Kelly (2004) classification scheme. It is very similar to the Steins spectral behaviour, even if the $0.49 \mu \mathrm{m}$ absorption band has a lower depth, probably indicative of a lower abundance of sulfides on its surface. Near-infrared spectra of 3103 Eger $(0.8-2.5 \mu \mathrm{m})$ were obtained by Gaffey et al. (1992), who did not report any specific mineral absorption feature, and by Clark et al. (2004), who found a shallow feature at $0.9 \mu \mathrm{m}$.

\section{Dynamical link between Steins and Eger}

Our data show that both Steins and Eger belong to the E[II] subgroup. As reported in Fornasier et al. (2007) in their survey of E-type asteroids, 8 bodies (64 Angelina, 434 Hungaria, 2035 Stearns, 2048 Dwornik, 2867 Steins, 3103 Eger, 4660 Nereus, and 6911 Nancygreen) are classified as E[II] subgroup members, due the presence in their spectra of the peculiar $0.49 \mu \mathrm{m}$ band. Four of them populate the Hungaria regions, 2 are NEOs (3103 Eger and 4660 Nereus), and 2 are main belt asteroids 
(2867 Steins, the Rosetta target, and 64 Angelina, the largest member). Inside the E[II] subgroup Steins and Eger show a very similar spectral behaviour, both in the spectral slope value, the highest one inside the subgroup, and in the depth of the $0.49 \mu \mathrm{m}$ band. Only 64 Angelina has a similar well pronunced $0.49 \mu \mathrm{m}$ band, with a depth of $8 \%$, but its spectral slope is significantly different $\left(4.06 \pm 0.61 \% / 10^{3} \AA\right)$ to Steins and Eger. The other members have fainter $0.49 \mu \mathrm{m}$ absorption bands (depth lower than 3-4\%) and spectral slope values always lower than $6 \% / 10^{3} \AA$ (Fornasier et al. 2007).

The spectral similarity between Steins and Eger is suggestive of a common origin in terms of mineralogical composition even if their orbits are significantly different. An intriguing possibility is that both Eger and Steins are former members of an old asteroid family outcome of the catastrophic disruption of a small parent body in the main belt. This link can be significantly reinforced by finding a dynamical pathway from the region where Steins is located and the present orbit of Eger. A first hint that this path exists come from the fact that Steins' orbit is very similar to that of 4 Vesta and we know that some Vesta "chips" have been found in Earth-crossing orbits (Bus \& Binzel 2002). The small members of a breakup event in the region of the main belt where both Vesta and Steins are located are rapidly dispersed by the Yarkovsky effect and resonances.

To test the possibility that some fragments of this Steins' family indeed drifted in semimajor axis by the thermal force entering resonances that drove them into the Earth-crossing region, we have explored the dynamical evolution of some clones of Steins. Since the drifting rate due to Yarkovsky drag scales almost inversely with the characteristic size $(1 / R)$, we expect that Steins, with a diameter between 4.8 and $6 \mathrm{~km}$ (Kuppers et al. 2007), which is about 4 times larger than Eger, is presently closer to the breakup location of the parent body. The higher Yarkovsky mobility of Eger possibly drove it into some resonance and then into the population of near-Earth objects. The parent body was probably an asteroid not much larger than Steins.

We then produced 40 clones of Steins representing the fragments of the family breakup. To all these bodies we assign a diameter of $1.5 \mathrm{~km}$, the present diameter of Eger (Veeder et al. 1989), and we follow their evolution under the influence of the gravitational force of the planets and of the Yarkovsky force. We used the numerical integrator SWIFT-RMVSY, the version of SWIFT modified by Broz (1999) to account for the diurnal and seasonal Yarkovsky effect. The bulk density of each body is set to $2.5 \mathrm{~g} / \mathrm{cm}^{3}$, and the density of the material at the surface is set to $1.5 \mathrm{~g} / \mathrm{cm}^{3}$. We adopted a value of $0.001 \mathrm{~W} / \mathrm{m}^{3} / \mathrm{K}$ for the thermal conductivity that, according to Morbidelli \& Vokroulicky (2003) is a reasonable value for small asteroids. The specific heat capacity $\mathrm{C}$ is set to $680 \mathrm{~J} /(\mathrm{kg} \mathrm{K})$ and the emissivity to 0.9 (Farinella et al. 1998). The albedo is assumed to be that of Eger and equal to 0.63 . The rotation period of all bodies is equal to that of Eger i.e. $5.7 \mathrm{~h}$ and we assume a random orientation of the spin axes in space. Different drift rates are then obtained depending on the obliquity. We included reorientation of drifting bodies due to collisions and YORP (Yarkovsky-O'Keefe-RadzievskiiPaddack effect) (Capek \& Vokrouhlick 2004).

As noted before, the orbit of Steins is close in the orbital element space to that of asteroid 4 Vesta and, as a consequence, we expect that the evolution of fragments from a putative Steins family closely matches that of Vesta family members (Carruba et al. 2005). Since the size of the Eger-type bodies is small, they are strongly affected by the Yarkovsky force and only major dynamical features, like the 3:1 mean motion resonance (MMR)

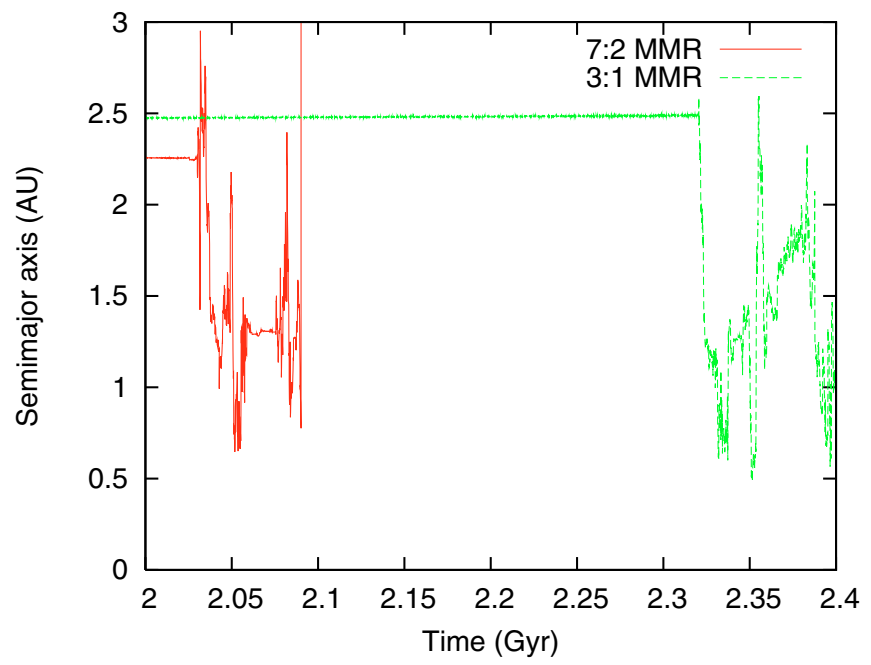

Fig. 2. Orbital evolution of two Steins clones injected into Earthcrossing orbits. One clone drifts outwards and enters the 3:1 MMR, while the other drifts inwards encountering the 7:2 MMR. Both the bodies, once in resonance, evolve rapidly into an Earth-crossing orbit.

with Jupiter at 2.5 AU or the 7:2 MMR with Jupiter at 2.256 AU (Carruba et al. 2005), can lead them into planet crossing orbits. Indeed several V-type asteroids have been found among NEAs (Bus \& Binzel 2002) showing that there is a dynamical pathway between the location of Vesta (and eventually Steins) and the near-Earth objects.

We have followed the evolution of these clones in time and found that, after $2.5 \mathrm{Gyr}$ of numerical simulation, 6 of the 40 fragments of the Steins family have reached the 3:1 resonance while 4 reached the 7:2. They enter a chaotic state and most of them spend some time as a NEO. In Fig. 2 we show the orbital evolution of two bodies being injected in the Earth-crossing region by the two resonances. These two cases well represent the possible evolution of Eger from the breakup of the Steins parent body until the present. From a dynamical point of view, the Steins family cannot be younger than about $2 \mathrm{Gyr}$, the timespan required by the first clone to become a NEA, otherwise we would not observe Eger. We do not have any constraint on the upper limit of the family age since we cannot reproduce the reorientation history of Eger's spin back in time.

An age of more than 2 Gyr for the family would significantly reduce the number of original fragments reaching the NEA region while driven by the Yarkovsky force. The collisional lifetime of $1 \mathrm{~km}$ sized bodies in the main belt is about $800 \mathrm{Myr}$, according to Bottke et al. (2005). As a consequence, only $10 \%$ of the original small family members would survive long enough to enter the 3:1 and 7:2 MMR ending up in the Earth region. Assuming that the family, just after the breakup, was made up of about $10^{3}$ fragments with a size comparable to that of Eger, we would expect that only about 100 of them would survive and become NEA asteroids at a rate of about one every 20 Myr according to our dynamical results. The median lifetime of the nearEarth objects population is about $10 \mathrm{Myr}$ (Gladman 2000) and, statistically, we should observe only one member of the Steins family per unit time within the NEA population. This rough reasoning seems to confirm the fact that only Eger among NEAs has a strong similarity to Steins. Of course, these computations are affected by strong uncertainties. The collisional lifetime of $1 \mathrm{~km}$-sized asteroids can easily change by more than a factor of ten if different values of the impact strenght of the bodies are 
assumed. Even a change in the poorly known slope of the main belt asteroid population at the small size end would lead to a significant change in the collisional lifetime. In addition, even the number of fragments from the breakup of an asteroid is poorly constrained, although steep slopes are expected among asteroid families (Tanga et al. 1999).

The present incompletness limit of the main belt asteroid population at small sizes might hide additional small family members in the process of becoming NEA. An important confirmation of the existence of a Steins family would be the detection of new small E-type asteroids between the present location of Steins and the two resonances.

\section{Discussion}

Many asteroid families have formed in the main belt because of cratering or breakup events. The most prominent families, like Themis or Eos, are easily recognizable, appearing as populous clusterings in the proper element space. Smaller ones may be undetectable, either because the completeness limit is too large, preventing the detection of most family members, or because they have been eroded and dispersed. The erosion is related to the subsequent collisional evolution of the family members involved in impacts with background asteroids (Marzari et al. 1995). The dispersal of small family members is due either to the chaotic evolution caused by the closeness to a resonance (Milani \& Farinella 1995) or to the low but steady migration by the Yarkovsky effect (Bottke et al. 2001). According to the simulations of the Marzari et al. (1999) modelling of the collisional evolution of the asteroid belt, there could have been about 800 breakup events over the solar system history involving a parent body in the size range between 10 and $20 \mathrm{~km}$ in diameter. This is approximately the size of the parent body of the Steins family. The collisional erosion and dynamical spreading of the family over 2 Gyr may have led to the present scenario, with only two family members known so far and orbiting in apparently separate regions of the belt.
Indeed, the Steins family formed in a dynamically active region where many resonances cause rapid chaotic evolution of the Yarkovsky drifting fragments. However, when the completeness limit of the asteroid population will be further lowered by small asteroid surveys like SKADS (Gladman et al. 2007), we may expect to find additional small families, possibly less dispersed than that of Steins if they formed in a less active dynamical environment.

\section{References}

Barucci, M. A., Fulchignoni, M., Fornasier, S., et al. 2005, A\&A, 430, 313 Barucci, M. A., Fornasier, S., Dotto, E., et al. 2007, A\&A, submitted Binzel, R. P. 1995, Meteoritics, 30, 486

Bottke, W. F., Vokrouhlicky, D., Broz, M., Nesvorny, D., \& Morbidelli, A. 2001, Science, 294, 1693

Bottke, W. F., Durda, D. D., Nesvorny, D., et al. 2005, Icarus, 175, 111

Broz, M. 1999, Diploma Thesis, Charles Univ., Prague, Czech Republic Burbine, T. H., Cloutis, E. A., Bus, S. J., Meibom, A., \& Binzel, R. P. 1998, Bull. Am. Astron. Soc., 30, 711

Bus, S. J., \& Binzel, R. P. 2002, Icarus, 158, 146

Capek, D., \& Vokrouhlicky, D. 2004, Icarus, 172, 526

Carruba, V., Michtchenko, T., Roig, F., Ferraz-Mello, S., \& Nesvorny, D. 2005, A\&A, 441, 819

Clark, B. E., Bus, S. J., Rivkin, A. S., et al. 2004, JGR, 109, 2001

Farinella, P., Vokrouhlicky, D., \& Hartmann, W. K. 1998, Icarus, 132, 378

Fornasier, S., \& Lazzarin, M. 2001, Icarus, 152, 127

Fornasier, S., Dotto, E., Marzari, F., et al. 2004, Icarus, 172, 221

Fornasier, S., Belskaya, I., Fulchignoni, M., Barucci, M. A., \& Barbieri, C. 2006, A\&A, 449, 9

Fornasier, S., Migliorini, A., Dotto, E., \& Barucci, M. A. 2007, Icarus, submitted Gaffey, M. J., Reed, K. L., \& Kelley, M. S. 1992, Icarus, 100, 95

Gaffey, M. J., \& Kelley, M. S. 2004, Lunar Plan. Sci. Conf., abs 1812

Gladman, B., Michel, P., \& Froeschle, C. 2000, Icarus, 146, 176

Gladman, B. J., Davis, D. R., Neese, C., et al. 2007, Icarus, in press

Kuppers, M., Mottola, S., Lowry, S. C., et al. 2007, A\&A, 462, 13

Marzari, F., Davis, D., \& Vanzani, V. 1995, Icarus, 113, 168

Marzari, F., Farinella, P., \& Davis, D. R. 1999, Icarus, 142, 63

Milani, A., \& Farinella, P. 1995, Icarus, 115, 209

Morbidelli, A., \& Vokrouhlicky, D. 2003, Icarus, 163, 120

Tanga, P., Cellino, A., Michel, P., et al. 1999, Icarus, 141, 65

Veeder, G. J., Hanner, M. S., Matson, D. L., et al. 1989, AJ, 97, 1211 